HNO 2011 · 59:1158-1159

DOI 10.1007/s00106-011-2337-5

Online publiziert: 1. Dezember 2011

(c) Springer-Verlag 2011

\author{
H.P. Zenner \\ Universitäts-Hals-Nasen-Ohren-Klinik, Tübingen
}

\title{
Alternative Therapieverfahren in der HNO-Heilkunde
}

die zeitliche - zur Maxime erhoben, diesen Anforderungen ist auch die medizinische Versorgung unterworfen. Andererseits gewinnen weiche Parameter wie etwa die zeitliche Zuwendung aus Sicht der Patienten zunehmend an Bedeutung in der Behandlung.

\section{$\checkmark$ Weiche Parameter wie die zeitliche Zuwendung gewinnen an Bedeutung} get, unbefriedigendes Ergebnis. Das Interesse am „Alternativen“ erscheint dann nur konsequent. Hier öffnet sich dem Therapeuten offenbar ein sehr weites Spektrum assoziierter Therapien. Die (traditionelle) chinesische Medizin nimmt hierbei eine Sonderstellung ein, die den besonderen Reiz für den wissenschaftlichen Mediziner zu erklären hilft. Basierend auf einer gewachsenen Philosophie stellt sie ein komplexes sich ergänzendes diagnostisches und therapeutisches System einer Erfahrungsmedizin dar. Außerhalb Chinas fand sicher die Akupunktur die weiteste Verbreitung. Interessanterweise greift die traditionelle chinesische Medizin in China selbst überwiegend auf die Arzneitherapie zurück. Weitere Säulen der traditionellen chinesischen Medizin Tuina (chinesische Manualtherapie) und Diätetik - sind in Deutschland kaum verbreitet, und Qigong wird weniger gezielt therapeutisch eingesetzt, sondern eher als Wellness- und Fitness-Training verstanden. Bei geschätzten 40.000 akupunktierenden Ärzten in Deutschland sollte man sich damit auseinandersetzen. Schließlich fragen auch Patienten vermehrt „alternative" Therapien nach. Einerseits wird in unserer Gesellschaft die Effizienz - auch
Nicht zuletzt dank der GERAC-Studie und der daraus resultierenden (extrabudgetären) Sonderstellung der Akupunktur hat sich diese Behandlungsform insbesondere in der Orthopädie zu einem wichtigen Standbein entwickelt. Die hierfür notwendigen Voraussetzungen für den Arzt sind neben einer Akupunkturausbildung auch der Nachweis über Kenntnisse in Schmerztherapie und Psychosomatik. Das hierbei vermittelte Verständnis über die Wirkung der Akupunktur entspricht dem einer Reflextherapie. Mit der philosophischen Gedankenwelt der traditionellen chinesischen Medizin hat dies nichts gemein.

Wer sich außerhalb der kassenärztlichen Indikationen bewegt, bedarf keiner zertifizierten Ausbildung. Vor diesem Hintergrund ist der ambitionierte Versuch von Herrn Greten (in diesem Heft), die traditionelle chinesische Medizin aus dem Reich der fernöstlichen Mystik in den klinischen Alltag zu rücken, sehr zu begrüßen.

Seinem Weg vom altchinesischen Orakel über Leibniz zu technischen Regelkreisen ist allerdings nicht leicht $\mathrm{zu}$ folgen. Die Latinisierung der chinesischen Begrifflichkeiten trägt in der anglophonen Medizin der Gegenwart nicht zwingend zum Erkenntnisgewinn bei. Auch wenn der Autor die Akupunktur GERAC-konform zunächst als Reflextherapie vorstellt, weist er jedoch recht bald auf die Nachteile eines derartigen Therapieverständnisses hin. Viel Wert wird deshalb auf die Einbettung der Medizin in die „harmonische“ Weltanschauung des traditionellen China gelegt und Disharmonien folgerichtig als Krankheitsverursacher herausgearbeitet. Neben dem weltanschaulichen Konzept werden klar strukturierte diagnostische Leitfäden vorgestellt, die zur Aufdeckung dieser „Disharmonien" führen.

Dass die Beobachtungen chinesischer Ärzte über physiologische und pathologische Vorgänge denjenigen ihrer europäischen Kollegen gleichen, ist dem gleichen beobachteten Subjekt - dem Menschen zu verdanken. Die Einordnung in ein entsprechendes gedankliches System als phänomenologische Beschreibung bzw. als deduktiver Schluss ändert nichts an dieser Gemeinsamkeit. Dass zur Beschreibung dieser beobachteten Vorgänge andere Termini verwendet werden, ist verständlich. Es ist ein dankenswerter Ansatz, diese durch Terminus und System unterschiedlichen Beschreibungen wieder auf den gemeinsamen Gegenstand der Betrachtung zurückzuführen.

Die chinesischen "Wandlungsphasen" kann man somit durchaus als vegetative Funktionsbeschreibungen interpretieren, was unserem physiologischen Verständnis entgegenkommt. Inwieweit eine 
Gleichsetzung der chinesischen Syndromdiagnostik als Parameter des „Neurovegetativums“, der „Mikrozirkulation“, des „Immunsystems“ bzw. der „Rolle des zellulären Funktionsgewebes“ gerechtfertigt ist, wird im vorliegenden Artikel leider nicht diskutiert. Allein der Ansatz trägt zu einem besseren Verständnis bei. Dass die Hals-Nasen-Ohren-Heilkunde ein Paradebeispiel für die Integration traditioneller chinesischer Medizin sein soll, erschließt sich nach der Lektüre allerdings nicht. Zwar beschäftigt sich das im Artikel erwähnte Standardwerk, das „Shang han lun“, mit Erkrankungen, die durch Kälte entstehen, doch sind damit - wie der Autor ausführt - keine Erkältungskrankheiten im westlichen Sinne gemeint. Der Artikel stellt somit den Versuch eines Überblicks über die zahlreichen Facetten der traditionellen chinesischen Medizin dar. Dabei werden deren Wurzeln erwähnt, eine Schematik der gedanklichen Struktur entworfen und eine Interpretation aus europäischer Sicht vorgenommen. Ein Vorgehen, das bereits Bände gefüllt hat und in der Kürze des Artikels sich nicht jedem Leser erschließen dürfte.

Die Vorgehensweise von Gleditsch (in dieser Ausgabe) erscheint vordergründig pragmatischer. Seine Jahrzehnte währende Erfahrung führt zu einer nahezu reflexhaften Auswahl von Akupunkturpunkten. Die zitierten subjektiven Behandlungserfolge sind jedem plausibel, der einmal das Vergnügen hatte, Herrn Gleditsch bei einer Behandlung zu erleben. Jeder Patient spürt sowohl die Aufrichtigkeit des Therapeuten als auch die Nadel. Die Leichtigkeit mit „very-point“ exakt den millimetergenau richtigen Punkt zu detektieren, ist allerdings nicht jedem gegeben. Als HNO-Arzt und Zahnarzt war es an diesem Autor, ein Akupunktur-Mikrosystem des Mundes zu entdecken und zu etablieren. Der Einsatz dieses Mikrosystems stellt für den nichtzahnärztlichen Therapeuten eine Herausforderung dar. Auch für den Patienten bedarf es einer gewissen Überwindung. Ein Artikel, der jedem Akupunkteur Ansporn sein sollte, es Herrn Gleditsch gleich zu tun. Die zitierte Tonsillektomie unter alleiniger Akupunktur-Analgesie ist jedoch als Einstieg ein sehr ehrgeiziges Ziel.
Die Arbeit von Schnitzler (in diesem Heft) geht wiederum andere Wege. Die chinesische "materia medica" stellt einen beachtlichen Wissensfundus über Arzneimittel, deren Wirkung und Nebenwirkung sowie mögliche Interaktionen dar. Eindrucksvoll wird dargelegt, dass derartige Arzneimittel eine substanzielle Bereicherung eines modernen Behandlungsregimes seien können. Es steht zu wünschen, dass sich neben der hier aufgeführten Behandlung der Herpesinfektion weitere Erkrankungen mit ähnlich potenten und nebenwirkungsarmen Kräutern therapieren lassen. Auch ist der Hinweis angebracht, das nicht nur Wirkstoffe aus chinesischen Kräutern, sondern sehr wohl auch aus einheimischen Pflanzen therapeutisch interessant seien können. Das Wissen über die Wirkung europäischer Kräuter ist allerdings bedeutend weniger stringent dokumentiert.
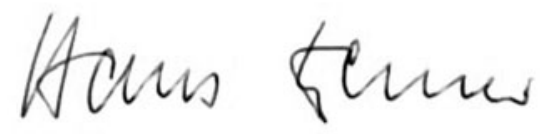

Prof. Dr. H.P. Zenner und Dr. S. Paasch, Tübingen

\section{Korrespondenzadresse \\ Prof. Dr. H.P. Zenner}

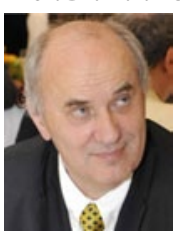

Universitäts-Hals-NasenOhren-Klinik Elfriede-Aulhorn-Str. 5 72076 Tübingen hans-peter.zenner@med.unituebingen.de

\section{Umfrageergebnisse zum Thema "Praxisabgabe"}

Die Mehrheit der niedergelassenen Ärzte blickt mit Angst auf den Verkauf ihrer Praxis, weil sie fürchten, keinen Nachfolger zu finden. Nur noch jeder vierte Arzt glaubt, dass er mehr als 100.000 Euro für seine Praxis erlösen kann; die Bedeutung des Praxisverkaufs für die Altersvorsorge relativiert sich dadurch. Das sind einige der auffälligsten Ergebnisse der Leserumfrage zum Thema Praxisabgabe, die die Fachverlagsgruppe SpringerMedizin und die Deutsche Apotheker- und Ärztebank initiiert haben. Mehr als 1000 Ärzte, darunter 700 Hausärzte und 300 Fachärzte, beteiligten sich an der Umfrage in der „Ärzte Zeitung“, im „WirtschaftsTipp“ und im Internet. Das Bild, das die Umfrage vermittelt, ist allerdings nicht durchweg negativ. So beurteilen Fachärzte die Situation deutlich entspannter: Hier sehen immerhin mehr als 40 Prozent die Praxisnachfolge als gesichert oder als wahrscheinlich an, und auch beim Praxiswert sind die Erwartungen höher als bei Hausärzten. Hinzu kommt, dass viele Ärzte auf die Schwierigkeiten reagieren, um so ihre individuelle Situation zu verbessern: Jeweils etwa ein Drittel will noch vor der Abgabe investieren, um die Praxis leichter verkaufen zu können. Ein weiteres Drittel der Umfrageteilnehmer will versuchen, über die Zusammenarbeit mit einem Juniorpartner einen Nachfolger zu finden. Und jeder sechste strebt an, die Praxis in eine Kooperation einzubringen.

Quelle: www.aerztezeitung.de (Suchwort: Praxisabgabe) 